\title{
The aesthetic fascination of the negative character. From expressionist monsters to Breaking Bad
}

\author{
La fascinación estética del personaje negativo. De los monstruos expresionistas a \\ Breaking Bad \\ Marcos Jiménez González \\ Universidad a Distancia de Madrid, España
}

\begin{abstract}
The representation of a negative character as the central axis of the story seems to have become popular since the premiere of Breaking Bad (2008-2013). The classic division between a protagonist who is a positive object of representation is followed by a story whose main character has negative narrative connotations. Despite having become widespread in recent years, it is a scheme that already existed in the cinema and in literature and that we, as viewers, have already seen in films of such a standing as The Godfather (1972), among others.

This lecture aims to glimpse the historical origin of the figure of the negative or perverse character in the cinema and, for this, we will go back to Europe at the beginning of the 20th century, in which serials such as Fantômas (1913) worked on the big screen and influenced the expressionist monsters of the 1920s, such as Dr. Caligari, Nosferatu or Dr. Mabuse.

By analyzing this cinema, the reasons why these characters arouse aesthetic fascination will be studied; In other words, the reasons why evil has aesthetic appeal in works of art will be investigated. Thus, we will ask ourselves why the Machiavellian characters are aesthetically admired, while morally they are rejected. This requires a reflection on the separation of aesthetic judgment and moral judgment, in the way that Schiller did, and the adaptation of that split to the present, making the aforementioned journey through the history of cinema.
\end{abstract}

Keywords: Cinema aesthetics, Expressionist monsters, Friedrich Schiller, The Godfather, Breaking Bad.

Introducción. Acercamiento práctico al problema: protagonistas, villanos y maestros del crimen

La distinción narrativa entre objetos positivos y objetos negativos de la representación es muy común en la literatura y en el cine. Todos nosotros entendemos con facilidad la diferencia entre un protagonista y un antagonista o entre un héroe y un villano que, construidos de forma binaria y asociados moralmente con la bondad y con la maldad, constituyen gran parte de la narrativa a la estamos acostumbrados. La identificación del espectador con el héroe y su admiración hacia él, contrastan fuertemente con el rechazo visceral que despierta en nuestra sensibilidad el villano, al que deseamos el destino trágico y propiamente negativo de la trama. Dicho proceso de identificación se produce en los esquemas narrativos clásicos, aquellos construidos sobre una división básica y maniquea de "buenos y malos" y que en el cine es típico del western clásico (indios y vaqueros), por ejemplo, o de las superproducciones dirigidas a un público muy amplio, que tienen pretensiones esencialmente comerciales: desde Die Nibelungen (Los nibelungos) (1924), de Fritz Lang, pasando por Star Wars (La guerra de las galaxias) (1977), de George Lucas, hasta llegar a las primeras partes de The Lord of the Rings (El señor de los anillos) (2001) o, incluso, de Harry Potter (2001). Todas ellas tienen en común un esquema narrativo que contrapone estética y moralmente a los personajes y en el que se orienta al espectador para que se posicione junto al héroe y contra el villano.

Pero ¿qué sucede cuando dicho esquema fracasa?; ¿qué ocurre cuando el eje central de la historia es el propio villano y no hay lugar en la narración para un héroe positivo? Esta es una cuestión sustancial que debemos abordar debido a la multitud de documentos en los que observamos este fenómeno. Como antecedente histórico, resulta interesante la creciente popularidad del género negro durante la década de los años treinta del siglo pasado; se trata de un estilo que en la literatura había aparecido varias décadas antes y que cobra protagonismo poco a poco en la gran pantalla. En películas como Underworld (La ley del hampa) (1927) o The Maltese Falcon (El halcón maltés) (1941) se detectan personajes y situaciones ambiguas que no se caracterizan por estar moralmente definidas, sino por todo lo contrario: por mostrar narrativamente el punto de vista del criminal o de su organización. Este es uno de los elementos más atractivos de este género. Algo parecido ha ocurrido históricamente con la figura del maestro del crimen que, teniendo una personalidad malévola y perversa, conforma en muchas ocasiones el eje narrativo central de las películas y de las novelas. Ubicados en el género negro, podemos hablar de The Night of the Hunter (La noche del cazador) (1955) como filme que gira en torno a la figura de un maestro del crimen perverso; sin embargo, este icono tan llamativo estéticamente apareció años antes en la gran pantalla y pervive en la actualidad. En las siguientes páginas se hará un recorrido histórico en el que se revisarán algunos de los personajes más retorcidos y maliciosos de la historia del cine, para explicar los motivos de su interesante atracción estética.

\section{Fundamentación estética}

El gusto por el elemento negativo de la representación ha sido un tema recurrente en Estética 
desde que, en los siglos XVIII y XIX, pensadores como Schlegel o Rosenkraz reflexionaran sobre la posibilidad de que existiera una atracción estética por lo feo o por lo moralmente rechazable; en otras palabras, desde que la belleza se dejó de entender en un sentido tradicional de corte platónico y se separó del ámbito de la moral. Kant supuso un acercamiento a esta escisión en su análisis sobre el sentimiento de lo sublime, pero no es hasta un siglo después cuando se acepta de un modo más extendido que el juicio estético y el moral van por caminos distintos. Actualmente dicha visión está consolidada y es un tema común en los ensayos sobre Arte y Estética. Escritos como Ante el dolor de los demás (2003), de Susan Sontag, o Por qué nos encantan los sociópatas (2016), de Adam Kotsko, parten de esta premisa para reflexionar sobre la experiencia estética que otorga el sufrimiento o que nace de los elementos negativos de la representación, pero estas cuestiones llevan vigentes más tiempo, siendo las reflexiones sobre el arte trágico algunas de las más relevantes.

Si nos centramos en la pregunta que da título al libro de Kotsko nos acercaremos más al tema que nos incumbe, pues el propio autor es consciente de que aquellos personajes que son moralmente detestables, como Eric Cartman, de la serie South Park (1997), o Homer, de The Simpson (Los Simpson) (1989), son precisamente los que más atraen al público, abriéndose de una forma evidente la brecha entre el juicio estético y el moral, del modo en el que lo advirtió Schiller. En su escrito Sobre lo patético, Schiller repara en que un mismo elemento puede ser valorado de forma distinta si se juzga moralmente o si se hace estéticamente. De hecho, llega a la conclusión de que estos dos juicios se basan en premisas opuestas, guiándose el primero por el deber y el segundo por la libertad:

De todo esto se desprende que los enjuiciamientos
moral y estético, muy lejos de servirse de apoyo uno
a otro, más bien se estorban mutuamente, porque le
confieren al ánimo dos direcciones opuestas; pues la
ley de la razón que la misma razón exige como jueza
moral no casa con la desvinculación a la que aspira
la imaginación como jueza estética. (Schiller 2013, 22).

Una vez señalada la escisión, Schiller repara en la atracción estética por los elementos negativos a la que se ha hecho referencia al principio, sosteniendo que algunos hechos indignos e innobles para la moral pueden conservar su valor estético o encumbrarlo, y con ello se hace una pregunta que supone el centro de nuestro análisis: “¿Cómo sucede si no que rechacemos con aversión al carácter tibiamente bueno y sigamos a menudo al especialmente malvado con admiración palpitante?”. (Schiller 2013, 25). El autor lo justifica mediante la libertad, ya que entendemos que la personalidad malvada actúa sin límites morales, mientras que alguien noble actuará de acuerdo con las reglas y convenciones estipuladas, por lo que no esperaremos de él "la posibilidad de la voluntad absolutamente libre". (Schiller 2013, 25).
Adam Kotsko también repara, como hemos visto, en la atracción estética del carácter malvado, sin embargo, no se apoya en la teoría de Schiller para hacerlo, a pesar de llegar a las mismas conclusiones. De este modo, menciona que los rasgos propiamente sociopáticos de los personajes son los que les hacen alcanzar el éxito en la ficción: "Lejos de ser los obstáculos que serían en la vida real, estos rasgos son precisamente los que permiten al sociópata fantástico conocer las mieles del éxito" (Kotsko 2016, 11). A pesar de que Kotsko utiliza una terminología distinta, se está refiriendo al mismo problema que planteaba Schiller. Mientras este habla de la diferencia entre el juicio moral y el estético, Kotsko establece una división entre "vida real" y "fantasía" o "ficción", más adaptada al debate de nuestro tiempo, pero fundamentada en el mismo fenómeno. Asimismo, uno hablará de "carácter malvado" y el otro de "sociópata" y, sin embargo, se estarán refiriendo a la mismo.

Algunos de los personajes en los que repara Kotsko, como Homer o Cartman, traspasan ya los límites del mal gusto y serían inconcebibles sin entender la experiencia de la posmodernidad. Los personajes que vamos a abordar en estas páginas se parecen a estos porque su éxito solo se produce si el espectador hace el ejercicio de discernir entre juicios y no concibe, por tanto, lo bueno como algo inseparable de lo bello. Se trata de aquellos personajes que encarnan el mal, no en un sentido desagradable u obsceno, sino en el de ser su representación metafórica. Ocupan siempre el lado negativo de la narración, pero son el eje central del relato, y eso es lo interesante. Su arquetipo simbólico es Mefistófeles: "el espíritu que siempre niega" (Goethe 1978, 33), y sus múltiples variantes han aparecido en la gran pantalla desde los albores del cine. Comencemos el recorrido.

\section{Los monstruos expresionistas: Caligari, Nosferatu y Mabuse}

En la historia del séptimo arte observamos con frecuencia esta figura, entre maquiavélica y perversa, que tiene unos rasgos psicopáticos muy definidos y que debido a sus actos criminales alcanza un reconocimiento estético amplio. Un ejemplo es la película The silence of the Lambs (El silencio de los corderos) (1991) y el personaje de Hannibal Lecter. Si analizamos narrativamente el filme, veremos que el objeto positivo de la representación es Clarice y Hannibal conforma el negativo. No obstante, su construcción es tan potente que acaba absorbiendo por completo la atención del espectador, y automáticamente se convierte en el centro del relato hasta cuando no está en la pantalla. La omnipresencia es justamente una característica de este tipo de personajes: toda la narración gira en torno a ellos y acaban dominando la orientación de la trama.

Uno de los periodos en los que más abundaron fue en el cine temprano de la República de Weimar, en los primeros años del cine expresionista alemán. Durante el periodo de entreguerras, el cine alemán se movió entre la pluralidad de estilos y la representación de 
temas y motivos que iban desde el reflejo del trauma que dejo la derrota de la guerra, envuelto en el caos y la desesperación, hasta la producción de filmes de alpinismo (Bergfilme) en los que se rememoraba el viaje identitario romántico de años atrás. De entre toda la variedad y riqueza que se encuentra en el cine de Weimar, vemos un tipo de películas cuyo tema central es la presencia de personajes maquiavélicos, en forma de maestros del crimen o vampiros, y que encuentran su fundamento simbólico en la figura de Mefistófeles.

Das Cabinet des Dr. Caligari (El gabinete del doctor Caligari) (1920) es el primer ejemplo de este grupo de películas alemanas, y ya en su argumento se observa la presencia de esta figura. El centro de la narración es un supuesto farandulero que presenta a un sonámbulo como espectáculo de feria, al que maneja a su voluntad. Lo que nadie sospecha es que el feriante tiene una doble vida, es el doctor Caligari (figura 1), que utiliza esa capacidad para cometer crímenes y asesinatos. El contenido no deja de ser una variación del tema de El doble (Doppelgänger), un hombre con dos facetas: alegre feriante, por un lado, y malévolo doctor, por otro. Sin embargo, con Caligari pasa exactamente lo mismo que se ha apuntado con anterioridad a propósito de Hannibal Lecter: es tan potente y sus acciones son tan extravagantes que acapara gran parte de la atención, convirtiéndose en omnipresente.

Caligari es el primero de una larga lista de personajes que Siegfried Kracauer denominó como "tiránicos" (Kracauer 1985), debido a su carácter malévolo y absorbente, además de por ser un antecedente visual de los sucesos históricos catastróficos y totalitarios que experimentaría Alemania años después. Asimismo, y desde una perspectiva más cinematográfica que sociológica, Vicente Sánchez-Biosca los bautizará como "demiurgos", en el sentido de que controlan y dominan la narración del filme, dejando en un plano secundario otros elementos importantes de las películas.

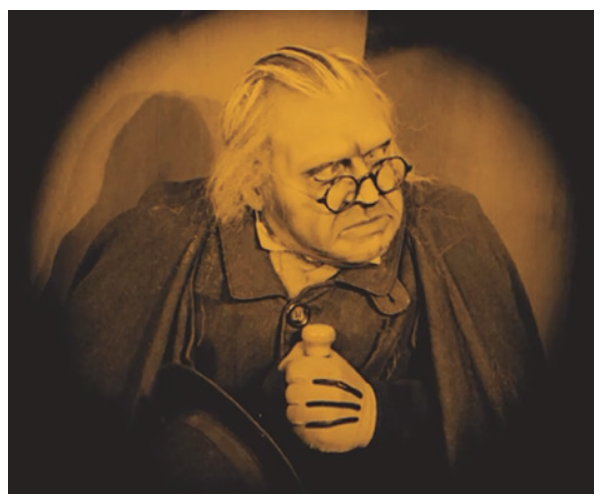

Figura 1 - Fotograma de Das Cabinet des Dr. Caligari Fuente: https://en.wikipedia.org/wiki/File:The_Cabinet_of_Dr_ Caligari_Werner_Krauss.png
Dos años después de Das Cabinet des Dr. Caligari se estrenan dos películas con personajes de este tipo: Nosferatu (1922) (figura 2) y Dr. Mabuse (El doctor Mabuse) (1922). En ambas, la figura principal es una encarnación del mal, heredera de Caligari y con los rasgos tiránicos mencionados; y, aunque hay diferencias entre ellos, existe una indudable influencia en cuanto a la construcción. Así lo advierte Sánchez-Biosca a propósito de la relación entre estos filmes:

Como Das Cabinet des Doktor Caligari, por ejemplo, Doktor Mabuse pone en escena un demiurgo que regula los designios del film. No obstante, media entre ambos films una sustancial diferencia: frente al de Wiene, el film de Lang no postula estructura delirante alguna ni por su desorden de lenguaje, propio de la paranoia, ni por la torturada figuración de sus imágenes. En el caso que sometemos a estudio ahora, la demiurgia de Mabuse se sitúa en un marco de transparencia narrativa bastante ajeno al que estábamos acostumbrados a ver sirviendo de marco a este modelo y tal aparición inesperada no puede resultar carente de contradicción respecto al funcionamiento de la demiurgia.

(Sánchez-Biosca 1990, 279).

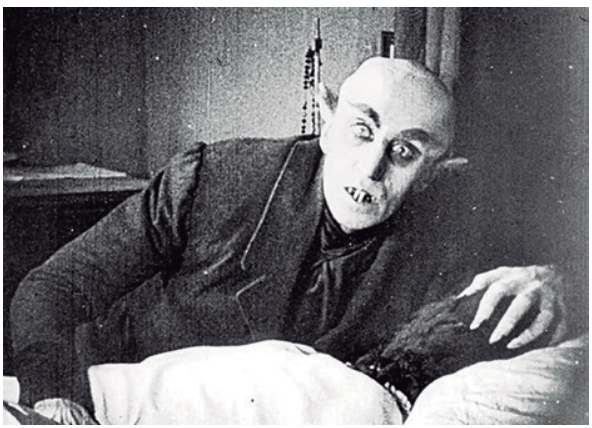

Figura 2 - Fotograma de Nosferatu (1922)

Fuente: https://www.libertaddigital.com/cultura/cine/2015-0715/la-leyenda-negra-de-nosferatu-1276552894/

El autor se refiere a que Mabuse no permanece en el ámbito de lo fantástico o de lo fantasmagórico, tal y como lo hacían Caligari o Nosferatu, sino que establece un puente entre la fantasmagoría típicamente expresionista y un caso real, ya que se trata de un maestro del crimen. En este sentido, es cierto que está inspirado en el serial francés Fantômas (1913-1914), de Louis Feuillade, cuyo centro narrativo es el maestro del crimen que da título a la serie. El serial influye en la película de Lang no solo en cuanto a la construcción de un personaje como demiurgo, sino también en cuestiones narrativas y estructurales del filme, cuestión que hemos tratado en la tesis Complejidad visual y narrativa en el cine de Fritz Lang en relación con el cine oscuro-expresionista alemán. (Jiménez González 2019, 172).

Este tipo de caracteres fueron propios del cine de Fritz Lang, tanto en la etapa alemana como en la estadounidense. En Europa, Mabuse (figura 
3) tiene sucedáneos en otras de sus películas. Si atendemos a Spione (Los espías) (1928), por ejemplo, encontraremos a Haghi (figura 4), un demiurgo de manual con los rasgos idénticos a Mabuse. Ambos son el eje de la narración debido a su omnipresencia, basada en que representan el Mal en su grado más alto. Otro personaje que puede incluirse en esta tipología es Hans Beckert, de $M$ - Eine Stadt sucht einen Mörder ( $M$, el vampiro de Düsseldorf) (1931), uno de los criminales más conocidos de la historia del cine.

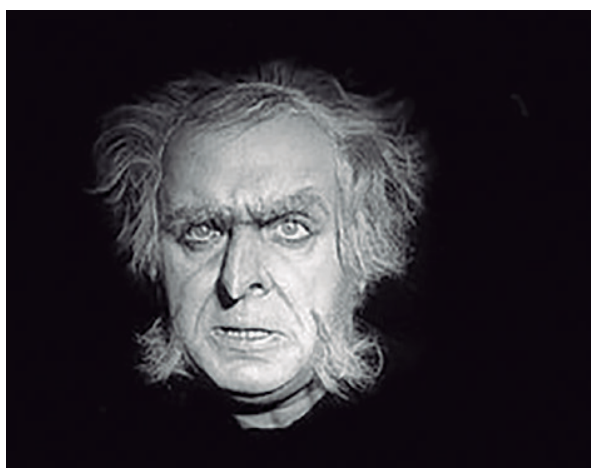

Figura 3 - Fotograma de Dr. Mabuse (1922)

Fuente: https://it.wikipedia.org/wiki/Dottor_Mabuse

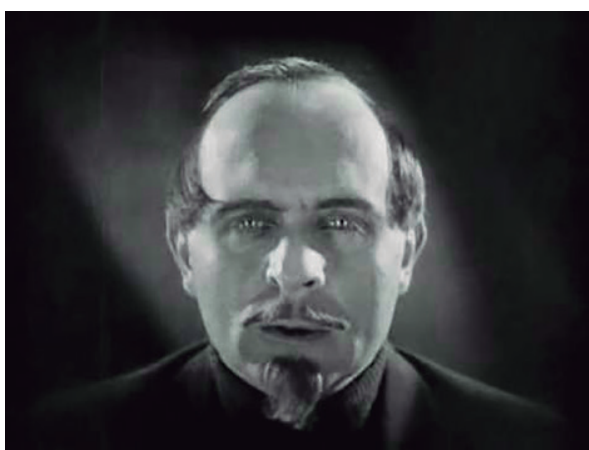

Figura 4 - Fotograma de Spione (1928).

Fuente: https://theartsdesk.com/film/dvd-spione

Aunque lo entendemos como una figura tiránica, Beckert tiene una construcción ambigua por ser verdugo y víctima al mismo tiempo, lo que hace que tanto él como la película alcancen una complejidad que no tenían las anteriores. Beckert es un asesino de niños al que todo el mundo busca para ajusticiarlo y su riqueza narrativa estriba en que no comete crímenes voluntariamente, sino movido por una fuerza maligna que lo posee. Por ello, el vampiro de Düsseldorf conforma dos polos opuestos que hasta entonces se habían representado por separado, porque en él se encuentran tanto Caligari como su principal víctima, Cesare. Se trata de un acercamiento al tema del doble, que será uno de los motivos recurrentes y favoritos de Fritz Lang a lo largo de toda su filmografía . De hecho, encontramos un personaje muy parecido a Hans Beckert en la película Secret Beyond the Door... (Secreto tras la puerta) (1947), que fue el que le condujo a escribir el texto ¿Por qué me interesa el asesinato? (1947). En este escrito Lang reflexiona sobre el atractivo estético del crimen y, entre otras cosas, se cuestiona el problema de la escisión que estamos tratando. En primer lugar, no entiende por qué a una "persona civilizada" puede gustarle el crimen en un sentido dramático, esto es, en la ficción, llegando a la conclusión de que uno de los motivos puede ser que la muerte es un elemento central de la dramaturgia y de la narrativa. Para justifícarlo se sirve de ejemplos de obras clásicas de Homero o de Shakespeare o recurre a la propia Biblia, teniendo en cuenta que todas ellas tienen la muerte como elemento sustancial del relato:

En la mayoría de los casos, dos horas de una vida humana representadas en la pantalla o en el escenario constituirían un espectáculo bien pobre. El drama empieza cuando se crea una fuerte emoción (...) Volvamos a Shakespeare (Hamlet, Macbeth, Otelo), a Homero o a la Biblia. La muerte es el argumento esencial del drama. (Lang 1947, citado en Eibel 1968, 132).

Esta reflexión de Lang lleva implícitas las mismas premisas que tenía Schiller cuando se preguntó por la admiración que profesamos hacia el carácter malvado y tiene las mismas connotaciones que los interrogantes que deja Adam Kotsko. El interés por el crimen se traduce aquí en el interés por el criminal, ya que en los tres escritos tenemos que hacer el ejercicio de separar el juicio estético del moral, además de entender que no solo actúan por separado, sino que en muchas ocasiones sus caminos son opuestos. Adaptándolo a una terminología actual, esto quiere decir que aquello que nos gusta en la ficción no tiene que corresponderse necesariamente con lo que nos satisface en nuestra vida cotidiana, y, de hecho, muchas veces va por líneas exactamente opuestas.

\section{El caso de Breaking Bad: Walter White contra Skyler}

A través de estos argumentos se ha hecho visible la potencia estética de los personajes negativos. Estableciendo un breve recorrido por algunas películas del expresionismo alemán se ha vislumbrado que aquello que nos repele moralmente, como un asesino o un crimen, puede satisfacernos en su faceta estética. Se han esclarecido los ejemplos de los criminales clásicos de la historia del cine, donde hay una distinción transparente entre ficción y realidad; sin embargo, existen algunos conflictos más recientes en los que, produciéndose esta separación, se logra un alto grado de identificación entre el espectador y el personaje negativo y, automáticamente, el positivo pasa a ser repudiado por el público en su valoración estética. El ejemplo de Breaking Bad (2008-2013) es muy apropiado para explicar dicho fenómeno. 
En esta serie, Walter White se sitúa en el centro de la narración, un profesor de carácter tímido que poco a poco se transforma en Heisenberg, uno de los narcotraficantes más importantes de Nuevo México. Esta transformación, que da título a la serie, es la que otorga a la historia el sentido tradicional del viaje del héroe, solo que esta vez ocurre a la inversa. White se trasforma en un objeto negativo de la representación, pero su carácter perverso le dota del atractivo estético propio de los maestros del crimen que hemos visto anteriormente. $Y$ es en este punto donde sucede un hecho importante respecto a la separación entre vida cotidiana y ficción o entre el juicio moral y el estético, que estamos tratando.

En el ámbito de la moral (vida cotidiana) White nos resultaría peligroso y lo rechazaríamos sin duda alguna. Entenderíamos que sus actos son ilegales y nos alejaríamos de él lo máximo posible; ahora bien, en su faceta estética (ficción) White nos interesa. De hecho, la lógica narrativa de la serie nos conduce a identificarnos con él y a rechazar todo aquello que aceptaríamos bajo el juicio moral (una familia estructurada, la legalidad, etc.). El hecho interesante es que esta experiencia del espectador es exactamente opuesta si atendemos al caso de Skyler, la mujer de Walter. Skyler es la figura que representa lo moralmente correcto, siendo un impedimento para que Walter potencie su faceta delictiva, esencia argumental e hilo conductor de la trama (figura 5). Esto desembocó en una crítica desorbitada del público hacia este personaje, mediante multitud de comentarios machistas y desagradables, que lo transformaron en el más odiado de la serie:

Estudiamos durante mucho tiempo los problemas que generaba el personaje de Walter y las dificultades para empatizar con él. En eso estábamos cuando de repente empezamos a ver que todos odiaban a Skyler, algo que todavía hoy me confunde. (Vince Gilligan en Héctor 2020).

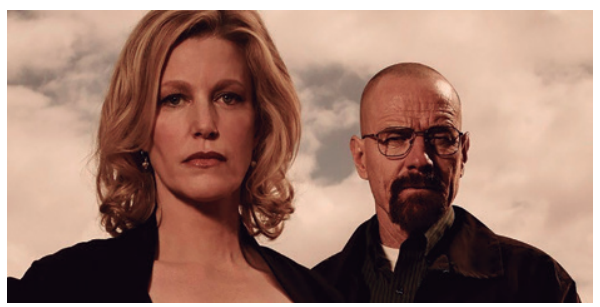

Figura 5 - Skyler y Walter White, de Breking Bad.

Fuente: https://www.fotogramas.es/series-tv-noticias/ a19470980/odio-skyler-breaking-bad-anna-gunn-declaraciones/

$\mathrm{Y}$, aunque las explicaciones de este odio son múltiples y sorprendentes para todo el reparto, lo cierto es que en el fondo se encuentra en la fascinación "visceral" que despertó Walter White en el público, que convertía en rechazable todo lo que se interpusiera en su camino. Así lo explicó Anna Gunn, la actriz que encana a Skyler:
La gente encontró un héroe en Walt, pero querían conectar con él tan visceralmente que ver a la persona que a menudo era su antagonista -por lo tanto, la antagonista de la serie de algún modo- les hizo sentir que ella estaba impidiéndole hacer lo que quería hacer y que debería permitírsele hacer lo que quería hacer (Anna Gunn en Elidrissi 2018).

Con el ejemplo de Walter y Skyler se observa a la perfección la gran diferencia existente entre los dos ámbitos que estamos tratando por separado. Walter es estético, mientras que Skyler es esencialmente moral; $y$, al ver un documento audiovisual o leer una novela, el público elige el camino estético por su potencia y por su fuerza, por ser un elemento liberador de su cotidianidad, que en muchas ocasiones le limita. Así lo expresa Schiller: "Dentro de los juicios estéticos [...] preferimos ver expresadas la fuerza y la libertad a costa de la ley racional antes que ver respetada la ley racional a costa de la fuerza y la libertad". (Schiller 2013, 24). En este sentido, Walter representa la fuerza y la libertad y Skyler la ley racional, y esta última funciona a la perfección en la lógica del juicio moral, pero es el primero quien domina la del estético.

\section{The Godfather: criminales estilizados, criminales ejemplares}

Las personalidades perversas tienen un gran atractivo y dejan en un plano secundario, si no en el antagonista, al resto de personajes del relato. Esto pasa con Walter, de Breaking Bad, pero también ocurría con los monstruos expresionistas alemanes de entreguerras y con las películas clásicas, entre el terror y el thriller, en las que abunda el recurso del carácter demiúrgico. Se ha mencionado el ejemplo de Hannibal Lecter, de The silence of the Lambs, pero esta narrativa también funciona en otras películas y lo hace de forma más sutil. Otro ejemplo es la saga de The Godfather (El padrino) (1972), donde ocurre exactamente lo mismo que en Breaking Bad: los personajes más despiadados, como el caso de Michael Corleone, son los más atractivos estéticamente (figura 6). De hecho, la evolución del personaje de Michael pasa por unas fases parecidas a las de Walter, ya que ambos se convierten en delincuentes, cuando previamente han partido del polo opuesto: Walter como profesor tímido e inocente y Michael como un soldado ejemplar de la marina, con un futuro prometedor en América, ubicado siempre dentro de la legalidad, cuyo objetivo es mantener limpio el apellido de la familia. De algún modo atraviesan un proceso de estetización, mediante el cual su comportamiento se concibe como ejemplar. Dicha estetización es la que explica que la saga The Godfather sea la que más guste a los mafiosos de verdad, tal y como afirma Íñigo Domínguez, periodista que ejerce de corresponsal en Roma para el periódico El correo. Domínguez afirma que los mafiosos admiran la película de Coppola, teniéndola como ejemplo. Personajes como Don Vito o Michael son arquetipos en los que los mafiosos se fijan y a partir de los cuales construyen su identidad. Estamos ante un ejemplo claro en el que el cine se alimenta de la realidad y 
viceversa; es decir, que en la formación de identidades y de modelos de comportamiento el cine y la vida se retroalimentan; debate importante en cualquier estudio sobre Estética del cine. En este caso, la mafia se sirve del modelo de la familia Corleone porque la encuentra elegante, estilizada y atractiva estéticamente, cosa que no ocurre con otras películas, como The Goodfellas (Uno de los nuestros) (1990), por ejemplo, ya que en ella se muestra el lado más amargo de las organizaciones criminales.

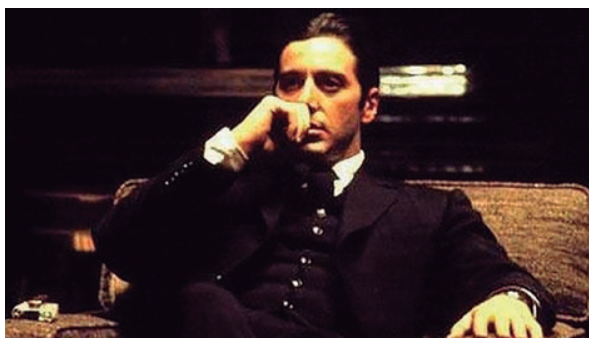

Figura 6 - Michael Corleone en un fotograma de The godfather. Part II.

Fuente; https://www.abc.es/play/cine/noticias/abci-sufrimientopacino-despues-rodar-padrino-terapia-cinco-veces-semanadurante-25-anos-201912300925_noticia.html

\section{Conclusiones}

A pesar de haber revisado varios tipos de personajes negativos y oscuros, y las razones de su fuerza estética, es cierto que existen diferencias entre ellos que enumeraremos a modo de conclusión.

En primer lugar, el atractivo de los monstruos expresionistas es puramente estético, fascinante, y supone casi una experiencia sublime para el espectador, ya que en su contemplación se diferencia claramente la ficción de la realidad. Esto es así porque se trata de películas fantasmagóricas, ubicadas en el género de terror, donde el público admite una posición distante desde el principio. Las figuras de Caligari, Mabuse o Nosferatu atraen nuestra atención más que ningún otro elemento del filme, pero no suponen un conflicto mayor que el de admitir que el personaje cruel es el que más nos gusta.

Si atendemos al segundo ejemplo explicado, el de Breaking Bad, observamos que hay una ligera variación, porque a la fascinación por el personaje negativo se le suma la identificación del espectador con él, cosa que ya desprende otras connotaciones. Aquí el público no experimenta el sentimiento de lo sublime, sino que se inmiscuye directamente en la trama y simpatiza con Walter hasta el punto de pretender que todos los aspectos de la trama estén a su favor; lo que conlleva, por supuesto, el odio visceral hacia Skyler y hacia todo lo que se interponga en su camino.

Por último, se ha revisado la identificación de la mafia con la familia Corleone en The Godfather. En este caso, a los fenómenos anteriores (fascinación y simpatía) se le suma su aplicación a la vida real, ya que la mafia se fija en las películas para construir sus modos de conducta. Para un espectador medio
The Godfather permanecería en el estadio propio de Breaking Bad; sin embargo, para la propia mafia, que se ve reflejada y embellecida, que se gusta a sí misma, supone un modelo para la vida cotidiana.

Tenemos entonces tres experiencias distintas, que suceden con la aparición del objeto negativo de la representación como centro indiscutible del relato y, dependiendo de la película a la que se atienda y del tipo de espectador que seamos, estaremos ante una u otra. Así, la sensación que nos produce The Silence of the Lambs podremos asociarla a la experiencia que nos otorgan las películas expresionistas de los años veinte, mientras que, si atendemos a la serie Narcos, la experiencia se asemejará más a la que tenemos con Breaking Bad.

Por tanto, el esquema narrativo amoral suscita unas sensaciones que no se daban en el binario (dividido en protagonistas y antagonistas) y exige al espectador un ejercicio más elaborado en el que se pone en tela de juicio su gusto y su código ético; cuestión que genera problemas e interrogantes difíciles de abordar desde una perspectiva simple de la realidad, cargada de maniqueísmo, teniéndose que admitir su fuerte complejidad.

\section{Bibliografia}

Eibel, Alfred. 1968. El cine de Fritz Lang. Traducido del francés por Tomás Segovia. México: Ediciones Era.

Elidrissi, Fátima. 2018. "¿Por qué los fans de Breaking Bad odiaban a Skyler?" El mundo, 9 de julho, 2018 https:// www.elmundo.es/television/2018/07/09/5b434816468aebf d398b4631.html.

Goethe, Johann Wolfgang von. 1978. Fausto, Madrid: Mediterráneo.

Héctor. 2020. “¿Por qué todos odiábamos a Skyler en "Breaking Bad"?" Alucine, un blog de cine, 23 de dezembro, $2020 \mathrm{https} / / / w w w . b o l s a m a n i a . c o m / c i n e / p o r-$ que-todos-odiabamos-a-skyler-en-breaking-bad/.

Jiménez González, Marcos. 2019. Complejidad visual y narrativa en el cine de Fritz Lang en relación con el cine oscuro-expresionista alemán. Tesis de doctoroado. Universidad de Salamanca.

Kotsko, Adam. 2016. Por qué nos encantan los sociópatas. Traducido del inglés por Albert Fuentes. Tenerife: Melusina.

Kracauer, Siegfried. 1985. De Caligari a Hitler; una historia psicológica del cine alemán. Traducido del inglés por Hector Grossi. Barcelona: Paidós.

Sánchez-Biosca, Vicente. 1990. Sombras de Weimar. Contribución a la historia del cine alemán 1918-1933, Madrid: Verdoux.

Schiller, Friedrich. 2013. Escritos breves sobre estética. Traducido del alemán por Juan Pablo Larreta y Víctor Borrero. Huelva: Gegner Libros.

Sontag, Susan. 2020. Ante el dolor de los demás. Traducido del inglés por Aurelio Major. Barcelona: Debolsillo.

\section{Filmografia}

Breaking Bad. 2008-2013. De Vince Gilligan. Estados Unidos: High Bridge Productions, Gran Via Productions, Sony Pictures Television. 
Das Cabinet des Dr. Caligari. 1920. De Robert Wiene. Alemania: Decla-Bioscop AG.

Die Nibelungen: Siegfried. 1924. De Fritz Lang. Alemania: Decla-Bioscop AG, Universum Film (UFA).

Dr. Mabuse. 1922. De Fritz Lang. Alemania: Uco-Film $\mathrm{GmbH}$.

Fantômas. 1913-1914). De Louis Feuillade. Francia: Société des Etablissements L. Gaumont.

Harry Potter and the Sorcerer's Stone. 2001. De Chris Columbus. Reino Unido/ Estados Unidos: Warner Bros., Heyday Films.

M. 1931. De Fritz Lang. Alemania: Nero-Film AG.

Star Wars. 1977. De George Lucas. Estados Unidos: Lucasfilm, Twentieth Century Fox.

The Godfather. 1972. De Francis Ford Coppola.

Estados Unidos: Paramount Pictures.

The Goodfellas. 1990. De Martin Scorsese. Estados Unidos: Warner Bros.

The Lord of the Rings: The Fellowship of the Ring. 2001.

De Peter Jackson. Estados Unidos: New Line Cinema, WingNut Films, The Saul Zaentz Company.

The Maltese Falcon. 1941. De John Huston. Estados Unidos: Warner Bros.

The Night of the Hunter. 1955. De Charles Laughton. Estados Unidos: Paul Gregory Productions.

The Silence of the Lambs. 1991. De Jonathan Demme. Estados Unidos: Strong Heart/Demme Production, Orion Pictures.

Underworld. 1927. De Josef von Sternberg y Arthur Rosson. Estados Unidos: Paramount Pictures. 